\title{
Circular Arc Approximation by Quartic H-Bézier Curve
}

\author{
Maria Hussain \\ Department of Mathematics \\ Lahore College for Women University, Pakistan \\ maria.hussain@lcwu.edu.pk \\ W. E. Ong \\ School of Mathematical Sciences \\ Universiti Sains Malaysia, Malaysia \\ weneng@usm.my \\ Farah Nazir \\ Department of Mathematics \\ University of the Punjab, Lahore, Pakistan \\ farah714@live.com \\ Malik Zawwar Hussain \\ Department of Mathematics \\ University of the Punjab, Lahore, Pakistan \\ malikzawwr.math@pu.edu.pk
}

\begin{abstract}
The quartic H-Bézier curve is used for the approximation of circular arcs. It has five control points and one positive real free parameter. The four control points are carried out by $G^{1}$-approximation constraints and the remaining control point is dividing the line segment joining the second and fourth control points in the ratio 1:2. Optimized value of free parameter $\alpha$ is obtained by minimizing the maximum value of absolute radius error of the recommended approximation scheme. The developed approximation scheme is found considerably better than the existing approximation schemes for these computed values of control points and optimized value of the free parameter.
\end{abstract}

Keywords: Quartic H-Bézier curve, Control points, Free parameter, $G^{1}$-approximation constraints, Absolute radius error.

AMS Subject Classification 2010: 65D17, 68U07

\section{Introduction}

Circles and circular arcs are widely applicable in the field of CAD for the designing of various objects. The most common applications can be seen in the designing of highway and railway routs and in the construction of suspension bridges (Lu, 2012). Since the designers can not directly use the parametric equations of circles in CAD rather they use the approximations of circles. So it is of keen interest of many authors to find the optimal approximations of circular arcs. Ahn and Kim (1997) used Bernstein-Bézier curves of degree four and five for $G C^{k}, k=2,3$, approximation of circular arcs. Order of approximation of these schemes was eight and ten respectively. Fang (1998) discussed five circular arcs approximation methods by using polynomial curves of degree 5. The convergence rate of these methods was either 8 or 10. Floater (1995) approximated the conic sections by quadratic splines with continuous curvature. Hur and Kim (2011) used 
cubic and quartic Bézier curves for the $G^{k}, k=1,2$, approximation of circular arcs. The Hausdorff distance between the approximating Bézier curves and circle was least for the proposed approximation schemes. Lee et al. (2006) presented the $G^{0}$-approximation of circular arc by Bézier curve of degree 2. In (Piegl and Tiller, 2003), the integral B-spline curve of appropriate degree was used as an interpolant for the approximation of circular arcs. The approximation was carried out by interpolating the derivatives at terminal points and a few interior points. Riškus (2006) approximated circular arc by using cubic Bézier curve. The proposed scheme was valuable in CAD system as it supported Bézier curve to interchange data through any data formats.

H-Bézier curves (Lee and Ahn, 2015) have hyperbolic basis functions. These basis functions are known as H-basis functions. H-Bézier curves preserve the favourable characteristics of ordinary Bézier curves like end points and end tangents interpolation property, partition of unity, invariance under affine transformation. H-Bézier curves give better smooth shape-preserving curves as compared to ordinary Bézier curves. In the proposed study, the circular arc approximation scheme by the quartic H-Bézier curve is presented. The existing approximation schemes of circular arcs use $G^{2}$-constraints for computing the control points of quartic and quintic approximating polynomials (Fang, 1998). Here the control points $b_{i}, i=0,1,3,4$, are evaluated by $G^{1}$-approximation constraints. The control point $b_{2}$ is chosen as a point dividing the line segment $\overline{b_{1} b_{3}}$ in the ratio $1: 2$. The value of free parameter $\alpha$ is evaluated by minimizing the maximum value of absolute radius error of approximation. The absolute radius error of the cultivated circular arc approximation scheme is considerably smaller than the existing schemes (Fang 1998; Lu 2012).

\section{Quartic H-Bézier Curve}

The quartic H-Bézier curve (Lee and Ahn, 2015)

$$
q(t)=\sum_{i=0}^{4} Z_{i}^{4}(t) b_{i}, t \in[0,1] .
$$

It has five control points $b_{i}, i=0,1,2,3,4$, a free parameter $\alpha(\alpha>0), t$ is H-Bézier parameter, and $Z_{i}^{4}(t), i=0,1,2,3,4$, are the quartic $H$-Basis functions given as:

$$
\begin{aligned}
& Z_{0}^{4}(t)=\frac{L}{A^{\prime}} Z_{1}^{4}(t)=\frac{R}{A}-\frac{M_{1}-M_{2}}{B C}, Z_{2}^{4}(t)=\frac{M_{1}-M_{2}}{B C}-\frac{N_{1}-N_{2}}{B C}, \\
& Z_{3}^{4}(t)=\frac{N_{1}-N_{2}}{B C}-\frac{Q}{A^{\prime}}, Z_{4}^{4}(t)=\frac{Q}{A^{\prime}}
\end{aligned}
$$

where

$$
\begin{aligned}
& \begin{array}{l}
L=\alpha^{2}\left(1+t^{2}\right)+2\left(1-\cosh \alpha(1-t)-2 \alpha^{2} t\right), A=\alpha^{2}+2-2 \cosh \alpha, \\
R=
\end{array} 2 \alpha^{2} t-\alpha^{2} t^{2}+2 \cosh \alpha(1-t)-2 \cosh \alpha, \\
& M_{1}=\left(\alpha^{2} t^{2}-2 \cosh \alpha(1-t)-2 \alpha t \sinh \alpha+2 \cosh \alpha\right)(\alpha \cosh \alpha+\alpha-2 \sinh \alpha), \\
& M_{2}=(\alpha-\sinh \alpha)\left(-2 \cosh \alpha(1-t)+\alpha^{2} t^{2} \cosh \alpha+\alpha^{2} t^{2}-2 \cosh \alpha t\right. \\
& \quad \quad+2 \cosh \alpha-2 \alpha t \sinh \alpha+2), \\
& N_{1}=-2 \cosh \alpha(1-t)+\alpha^{2} t^{2} \cosh \alpha+\alpha^{2} t^{2}-2 \cosh \alpha t-2 \alpha t \sinh \alpha+2, \\
& N_{2}=\left(\alpha^{2} t^{2}-2 \cosh \alpha t+2\right)(\alpha \cosh \alpha+\alpha-2 \sinh \alpha), Q=\alpha^{2} t^{2}-2 \cosh \alpha t+2,
\end{aligned}
$$




$$
B=\alpha \cosh \alpha+\alpha-2 \sinh \alpha, C=2 \cosh \alpha-2-\alpha \sinh \alpha .
$$

Numerical experiments suggest that the basis functions are either positive or negative depending upon the value of $\alpha$.

\section{Approximation of Circular Arc by Quartic H-Bézier Curve}

In this section, the numerical approximation scheme of circular arc by quartic H-Bézier curve is computed. The circular arc is considered in standard position i.e. center at origin $O(0,0)$, radius $r$, the initial point of the arc is along the positive horizontal axis and the final point is making a counter clockwise angle $\theta$ with the same axis. Any circular arc with arbitrary center can be transformed to this position by affine transformations.

The following $G^{1}$-approximation constraints are used for the approximation:

$$
\begin{aligned}
& \left.q(t)\right|_{t=0}=c_{0},\left.q(t)\right|_{t=1}=c_{1}, \\
& T_{0}=t_{0}, T_{1}=t_{1} .
\end{aligned}
$$

Here, $c_{i}{ }^{\prime} s$ are the end points and $t_{i}{ }^{\prime} s$ are the end unit tangent vectors of the circular arc. By using (1), we have $q(0)=b_{0}$ and $q(1)=b_{4}$. The end unit tangents of quartic $\mathrm{H}$ Bézier curves are denoted by $T_{0}$ and $T_{1}$ and are computed by the relation $T_{i}=\frac{q^{\prime}(i)}{\left\|q^{\prime}(i)\right\|}$, for $i=0,1$, which implies $T_{0}=\frac{b_{1}-b_{0}}{\gamma_{1}}$ and $T_{1}=\frac{b_{3}-b_{2}}{\gamma_{2}}$. Now by using these values of end points and end unit tangents of the quartic H-Bézier curve into (2) and (3) respectively, the following equations are obtained

$$
\begin{aligned}
& b_{0}=c_{0}, b_{4}=c_{1}, \\
& \frac{b_{1}-b_{0}}{\gamma_{1}}=t_{0}, \quad \frac{b_{4}-b_{3}}{\gamma_{2}}=t_{1} .
\end{aligned}
$$

The values of $\gamma_{1}=\left\|b_{1}-b_{0}\right\|$ and $\gamma_{2}=\left\|b_{4}-b_{3}\right\|$ are positive real numbers. The end points and the end unit tangents of the concerned circular arc $c_{0} c_{1}$ are $c_{0}(r, 0)$, $c_{1}(r \cos \theta, r \sin \theta), t_{0}(0,1), t_{1}(-\sin \theta, \cos \theta)$. Substituting these values in (4) and (5), the control points of quartic H-Bézier curve are given by

$$
\left.\begin{array}{c}
b_{0}=(r, 0), b_{1}=\left(r, \gamma_{1}\right), b_{2}=\left(b_{20}, b_{21}\right), \\
b_{3}=\left(b_{30}=r \cos \theta+\gamma_{2} \sin \theta, r \sin \theta-\gamma_{2} \cos \theta\right), b_{4}=(r \cos \theta, r \sin \theta)
\end{array}\right\}
$$

The values of parameters $\gamma_{1}$ and $\gamma_{2}$ are assumed as:

$$
\gamma_{1}=\left\|b_{1}-b_{0}\right\|=\frac{\left\|b_{4}-b_{0}\right\|}{4}, \gamma_{2}=\left\|b_{4}-b_{3}\right\|=\frac{\left\|b_{4}-b_{0}\right\|}{4} \text {. }
$$

It gives, $\quad \gamma_{1}=\gamma_{2}=\frac{\sqrt{(r \cos \theta-r)^{2}+(r \sin \theta)^{2}}}{4}$.

In (6), the control point $b_{2}$ can be evaluated by various methods. In this study, $b_{2}$ is chosen as a point which divides the line segment $\overline{b_{1} b_{3}}$ in the ration $1: 2$. The coordinates of $b_{2}$ are $b_{20}=\frac{r \cos \theta+\gamma_{2} \sin \theta+2 r}{3}, b_{21}=\frac{r \sin \theta-\gamma_{2} \cos \theta+2 \gamma_{1}}{3}$. 
In (1) by substituting the values of control points from (6), the following parametric equations of quartic H-Bézier curve are obtained:

$$
\begin{aligned}
& x(t)=Z_{0}^{4}(t) x_{0}+Z_{1}^{4}(t) x_{1}+Z_{2}^{4}(t) x_{2}+Z_{3}^{4}(t) x_{3}+Z_{4}^{4}(t) x_{4}, \\
& y(t)=Z_{0}^{4}(t) y_{0}+Z_{1}^{4}(t) y_{1}+Z_{2}^{4}(t) y_{2}+Z_{3}^{4}(t) y_{3}+Z_{4}^{4}(t) y_{4},
\end{aligned}
$$

where

$$
\begin{aligned}
& x_{0}=r, x_{1}=r, x_{2}=\frac{r \cos \theta+\gamma_{2} \sin \theta+2 r}{3}, x_{3}=r \cos \theta+\gamma_{2} \sin \theta, x_{4}=r \cos \theta, \\
& y_{0}=0, y_{1}=\gamma_{1}, y_{2}=\frac{r \sin \theta-\gamma_{2} \cos \theta+2 \gamma_{1}}{3}, y_{3}=r \sin \theta-\gamma_{2} \cos \theta, y_{4}=r \sin \theta .
\end{aligned}
$$

The H-basis functions $Z_{i}^{4}(t), i=0,1,2,3,4$, have been already defined in Section 2 . The free parameter $\alpha$ of quartic H-Bézier curve can assume different values and produces different H-Bézier curves for the approximation of circular arc. Therefore to find optimal approximation the value of $\alpha$ must be optimized. Here, the optimized value of $\alpha$ is obtained by the following optimization problem-I.

\section{Optimization problem-I:}

$$
\min _{\alpha>0}\left(\max _{0 \leq t \leq 1} \tilde{r}(\alpha, t)\right),
$$

subject to

$$
\alpha \geq u,
$$

where, $u=2.2204 \times 10^{-16}, \tilde{r}(\alpha, t)=\left|x^{2}(t)+y^{2}(t)-r^{2}\right| . \quad \tilde{r}(\alpha, t)$ is the absolute radius error of the developed approximation scheme for circular arc $c_{0} c_{1}$ by quartic $\mathrm{H}$ Bézier curve (1). $r$ is the radius of the concerned circular arc, $x(t)$ and $y(t)$ are defined in (8) and (9).

The optimization problem-I is solved by the MATLAB 7 built in function fminimax of the MATLAB optimization toolbox. The fminimax is based on the sequential quadratic programming technique (Brayton et al., 1979). Sequential quadratic programming technique is not suitable for discontinuous functions. But the objective function of optimization problem-I is continuous, so the problem is solvable.

\section{Numerical Example}

In this section, the numerical approximation scheme introduced in Section 3 is implemented on the unit circular arc given in Table 1.

Table 1: Unit circular arc

\begin{tabular}{|c|c|c|c|c|c|}
\hline $\boldsymbol{\theta}$ & $\boldsymbol{r}$ & $\boldsymbol{c}_{\boldsymbol{0}}$ & $\boldsymbol{c}_{\boldsymbol{1}}$ & $\boldsymbol{t}_{\mathbf{0}}$ & $\boldsymbol{t}_{\mathbf{1}}$ \\
\hline$\pi / 4$ & 1 & $(1,0)$ & $(0.7071,0.7071)$ & $(0,1)$ & $(-0.7071,0.7071)$ \\
\hline
\end{tabular}


Control points of the quartic H-Bézier curve corresponding to the unit circular arc of Table 1 are calculated by Theorem 1 . These computed values of control points and free parameter $\alpha$ are given in Table 2 .

Table 2: Control points of the quartic H-Bézier curve

\begin{tabular}{|c|c|c|c|c|c|}
\hline $\boldsymbol{b}_{\mathbf{0}}$ & $\boldsymbol{b}_{\mathbf{1}}$ & $\boldsymbol{b}_{\mathbf{2}}$ & $\boldsymbol{b}_{\mathbf{3}}$ & $\boldsymbol{b}_{\mathbf{4}}$ & $\boldsymbol{\alpha}$ \\
\hline$(1,0)$ & $(1,0.1913)$ & $(0.9475,0.3182)$ & $(0.8424,0.5718)$ & $(0.7071,0.7071)$ & 9.2149 \\
\hline
\end{tabular}

Graph of quartic H-Bézier curve approximating the circular arc of Table 1 is plotted in Figure 1. The reflection of this circular arc about the line $y=x$ is given in Figure 2. By combining the graphs of Figures 1 and 2, a quarter circle of unit radius is obtained ( Figure 3). A semi-circle is obtained in Figure 4 by the reflection of the quarter circle of Figure 3 about y-axis. Complete circle is obtained in Figure 5 by the reflection of semicircle of Figure 4 about $x$-axis. The maximum value of the absolute radius error of the developed approximation scheme is $8.7 \times 10^{-3}$. The plot of absolute radius error of the developed quartic H-Bézier approximation scheme is given in Figure 6.

\section{Conclusion}

In the proposed study, a circular arc quartic H-Bézier curve approximation scheme is introduced. The values of control points are evaluated by $G^{1}$-approximation constraints and the value of free parameter is obtained by minimizing the maximum value of absolute radius error of proposed approximation scheme. Absolute radius error of approximation for the proposed scheme is compared to the prevailing schemes (Table 3). It is noted that the absolute radius error of approximation in the proposed scheme is less than (Fang, 1998; Lu 2012).

\section{Table 3: Absolute radius errors}

\begin{tabular}{|c|c|c|}
\hline References & Methods & Absolute radius error \\
\hline Fang, 1998 & Fang method IV & $1.1788 \times 10^{-2}$ \\
\hline Lu, 2012 & Lu & $1.5213 \times 10^{-2}$ \\
\hline Section 4 & $\begin{array}{c}\text { Quartic H-Bézier approximation scheme } \\
\text { for circular arc }\end{array}$ & $8.7 \times 10^{-3}$ \\
\hline
\end{tabular}




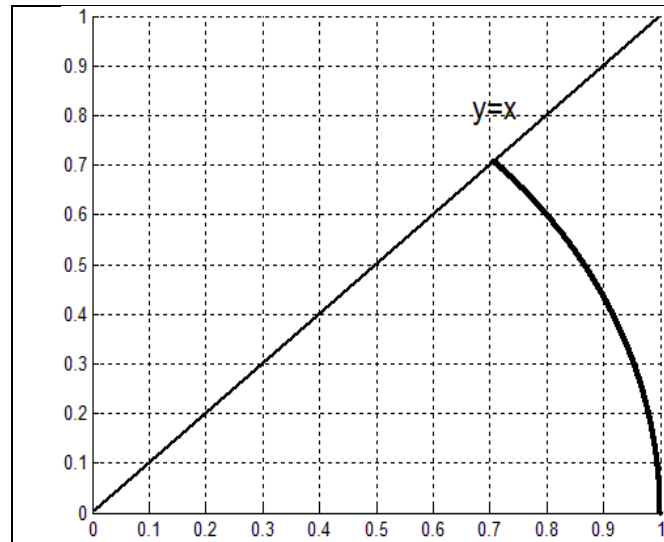

Figure 1: Unit circular arc making angle $\frac{\pi}{4}$ with $\mathrm{x}$-axis

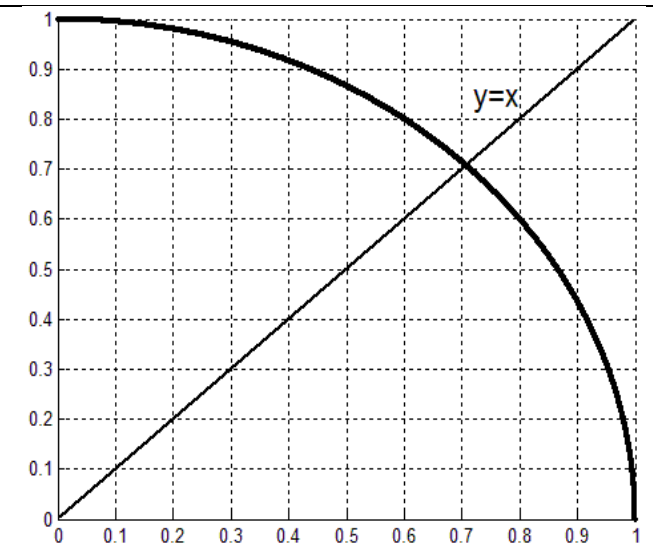

Figure 2: Reflection of Fig. 1 about the line $y=x$

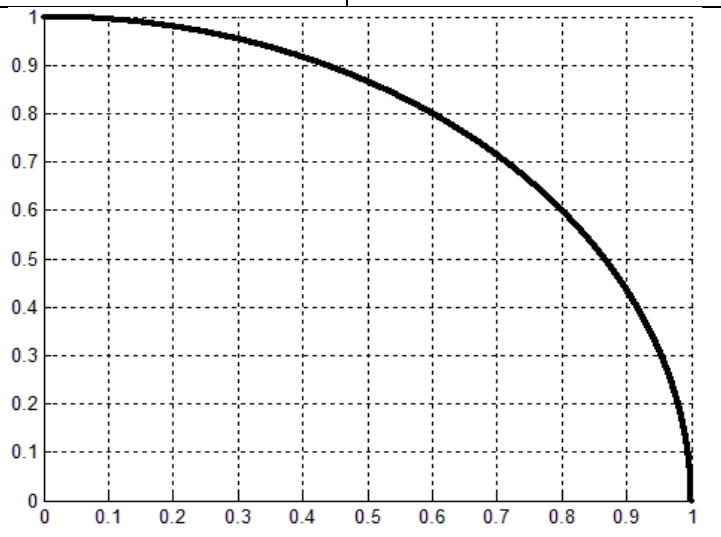

Figure 3: Combination of Figures 1 and 2

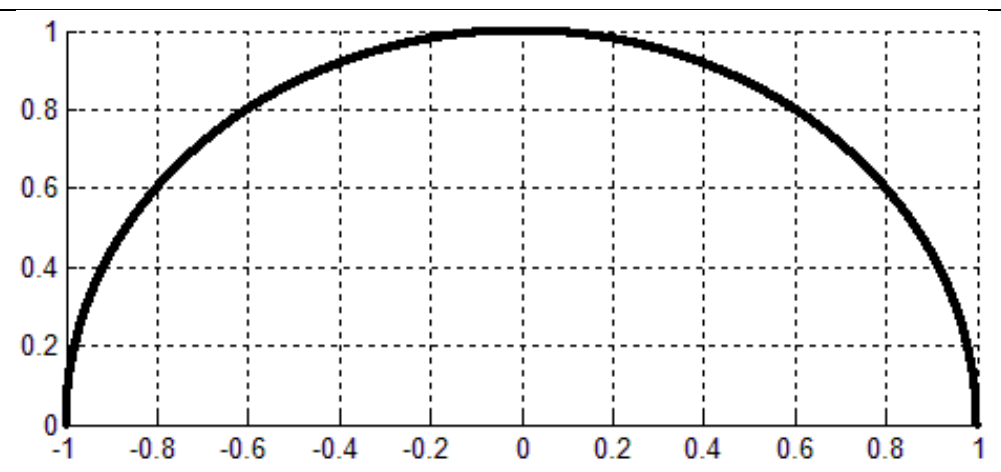

Figure 4: Reflection of Figure 3 about y-axis 


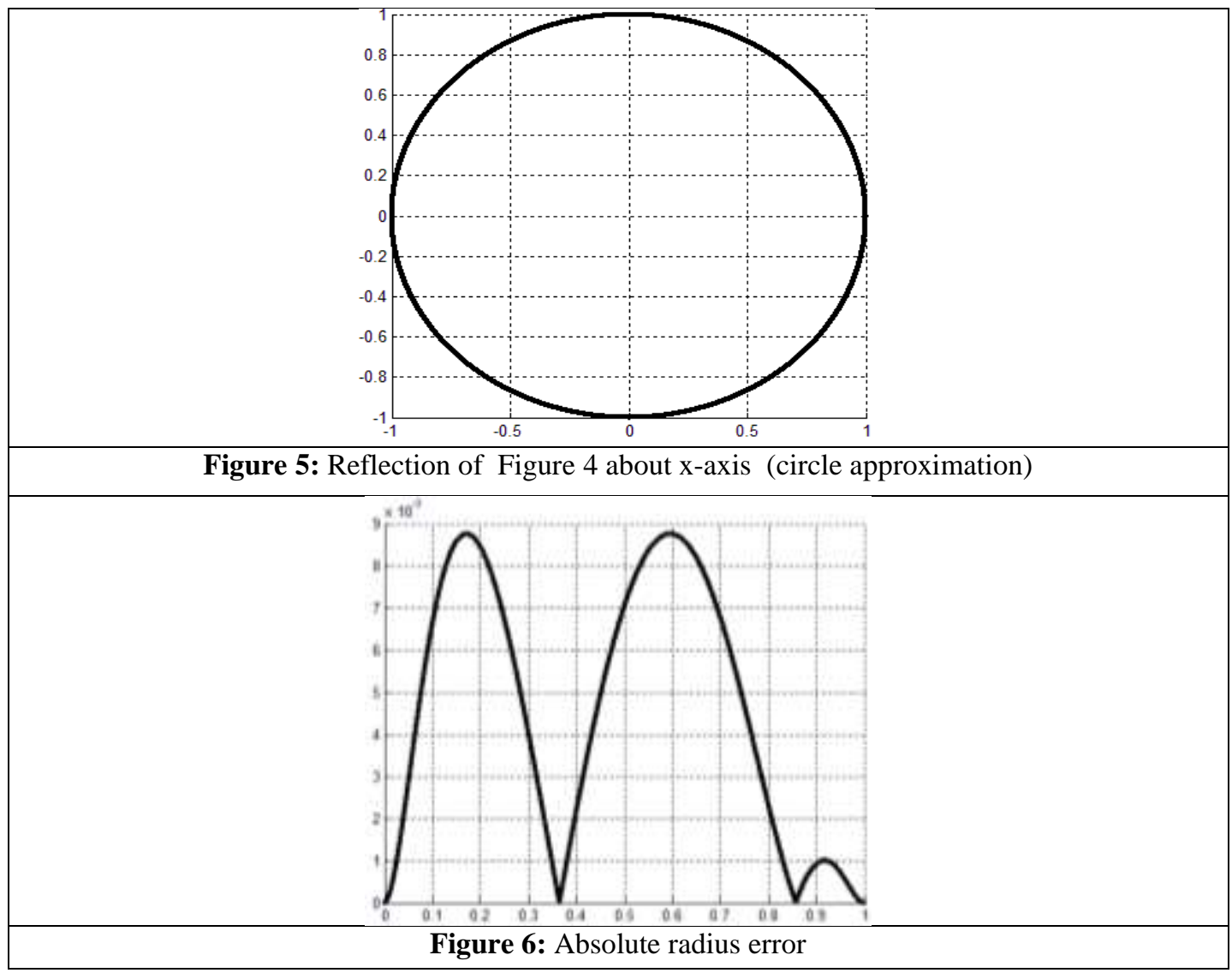

\section{Acknowledgements}

Malik Zawwar Hussain acknowledges Universiti Sains Malaysia for providing opportunity to carry out his part of this research at Universiti Sains Malaysia as a visiting professor.

\section{References}

1. Ahn, YJ. and Kim, H.O. (1997). Approximation of circular arcs by Bézier curves, Journal of Computational and Applied Mathematics, 81, 145-163.

2. Brayton, R.K., Director, S.W., Hachtel G.D. and Vidigal, L. (1979). A new algorithm for statistical circuit design based on Quasi-Newton methods and function splitting, IEEE Transactions on Circuits and Systems, 26, 784-794.

3. Fang, L. (1998). Circular arc approximation by quintic polynomial curves, Computer Aided Geometric Design, 15, 843-861.

4. Floater, M. (1995). High order approximation of conic sections by quadratic splines, Computer Aided Geometric Design, 12, 617-637.

5. Hur, S. and Kim, T.-w. (2011). The best G1 cubic and G2 quartic Bézier approximations of circular arcs, Journal of Computational and Applied Mathematics, 236, 1183-1192. 
6. Lee, I.K., Kim, M.S. and Elber, G. (1996). Planar curve offset based on circular approximation, Computer Aided Design, 28(8), 617-630.

7. Lee, R. and Ahn, Y.J. (2015). Limit curve of H- Bézier curves and rational Bézier curves in standard form with same weight, Journal of Computational and Applied Mathematics, 281, 1-9.

8. Lin, J., Ball, A.A. and Zheng, J.J. (2001). Approximating circular arcs by Bézier curves and its application to modeling tooling for FE forming simulations, International Journal of Machine Tools \& Manufacture, 41, 703-717.

9. Lu, L. (2012). On polynomial approximation of circular arcs and helices, Computers and Mathematics with Applications, 63, 1192-1196.

10. Piegl, L. and Tiller, W. (2003). Circle approximation using integral B-Splines, Computer Aided Design, 35, 601-607.

11. Riškus, A. (2006). Approximation of a cubic Bézier curve by circular arcs and vice versa, Information Technology and Control, 35(4), 371-378. 